\title{
Impacto del $\mathrm{CO}_{2}$ sobre la densidad celular en seis cepas de microalgas marinas
}

\section{Impact of $\mathrm{CO}_{2}$ on cell density in six marine microalgae strains}

\author{
Impacto do $\mathrm{CO}_{2}$ na densidade celular em seis \\ linhagens de microalgas marinhas
}

\author{
Alberto I. Oscanoa Huaynate*; Gheraldine A. Ynga Huamán; lliana L. Chang Ávila; \\ Carla P. Aguilar Samanamud
}

\begin{abstract}
Laboratorio de Biotecnología Acuática, Área Funcional de Investigaciones en Acuicultura, Dirección General de Investigaciones en Acuicultura, Instituto del Mar del Perú (IMARPE), Esquina Gamarra y General Valle S/N Chucuito, Callao, Perú.

*aoscanoa@imarpe.gob.pe
\end{abstract}

Fecha Recepción: 31 de marzo de 2015 Fecha Aceptación: 19 de junio de 2015

\begin{abstract}
Resumen
Debido a la gran facilidad con que las microalgas pueden capturar el $\mathrm{CO}_{2}$ del medio ambiente, resulta interesante evaluar la cantidad y tiempo de ingreso de éste a los cultivos masivos, con la finalidad de aumentar la densidad celular. El objetivo del presente estudio fue evaluar los tiempos de inyección del mencionado gas, durante la producción de biomasa que conlleve a una mayor densidad celular, evaluando además, la variación del pH sin alterar la calidad del cultivo. A partir de seis cepas obtenidas del Banco de Germoplasma del Instituto del Mar del Perú, se realizaron cultivos tipo batch de 300L en invernadero, el tiempo de cultivo de la fase exponencial donde se realizaron las pruebas fue de tres días. Los datos se procesaron mediante el análisis del parámetro pendiente de la regresión lineal. Los resultados mostraron que la densidad celular es inversamente proporcional al tiempo de inyección de $\mathrm{CO}_{2}$ al cultivo. La mayor densidad celular, en las diferentes cepas, se obtuvo a los $5 \mathrm{~min}$, excepto para las cepas Chaetoceros gracilis y Nannochloris maculata, las cuales obtienen la mayor densidad a los 10 y $15 \mathrm{~min}$, respectivamente. La variación de $\mathrm{pH}$ tendió hacia la acidez, en un rango de 8 a 4, sin alterar la densidad celular, por el contrario, los cultivos permanecieron libres de contaminantes. En conclusión, los resultados permiten establecer tiempos adecuados de inyección del $\mathrm{CO}_{2}$, los cuales fortalecen la fase de crecimiento exponencial aumentando la densidad poblacional en un $30 \%$ sobre lo establecido en esta fase.
\end{abstract}

Palabras clave: microalgas, densidad celular, $\mathrm{CO}_{2}$, cultivo masivo.

\begin{abstract}
As microalgae can capture $\mathrm{CO}_{2}$ easily from the environment, it is interesting to measure the amount and time control of the entry of this gas into microalgae mass culture, in order to increase cell density. The aim of this study was to evaluate the injection times of $\mathrm{CO}_{2}$ for biomass production that may lead to a higher cell density, it was also evaluated the $\mathrm{pH}$ variation without altering the quality of the crop. The work was made with six strains from the Germplasm Bank of the Instituto del Mar del Perú. There were performed cultures like $300 \mathrm{~L}$ batch in a greenhouse, the cultivation time of the exponential phase lasted three days. The slope of the regression line parameters was analyzed to process data. The results showed that the cell density is inversely proportional to $\mathrm{CO}_{2}$ injection time cultivation. The higher cell density
\end{abstract}

Cita: Oscanoa Huaynate Al, Ynga Huamán GA, Chang Ávila IL, Aguilar Samanamud CP. Impacto del CO sobre la densidad celular en seis cepas de microalgas marinas. rev.ion. 2015;28(2):23-32. 
was obtained after $5 \mathrm{~min}$ in different strains, except for strains Chaetoceros gracilis and Nannochloris maculate. Those microalgae got the highest density at 10 and $15 \mathrm{~min}$, respectively. The variation of $\mathrm{pH}$ tended toward acidity in a range from 8 to 4 , without altering the cell density and the cultures remained free of contaminants. In conclusion, the results can establish the appropriate times of $\mathrm{CO}_{2}$ injection, which strengthen the exponential growth phase by increasing population density in $30 \%$ over the usual results of this phase.

Keywords: microalgae, cell density, $\mathrm{CO}_{2}$, massive culture.

\section{Resumo}

Devido à facilidade com a qual as microalgas podem capturar $\mathrm{CO}_{2}$ do meio ambiente, é interessante avaliar a quantidade e o tempo da entrada deste no cultivo massivo, a fim de acrescentar a densidade celular. $\mathrm{O}$ objetivo deste estudo foi avaliar o tempo de injeção do referenciado gás na produção de biomassa que leva a uma maior densidade celular, avaliando a variação do $\mathrm{pH}$ sem prejudicar a qualidade do cultivo. A partir de seis linhagens obtidas do Banco de Germoplasma do Instituto del Mar del Perú, realizaram-se cultivos do tipo batch de $300 \mathrm{~L}$ em casa de vegetação, o tempo de cultivo da fase exponencial, onde foram realizados os testes, foi de três dias. Os dados foram processados através da análise do parâmetro de inclinação da regressão linear. Os resultados mostraram que a densidade de células tem uma relação negativa com o tempo de injeção de $\mathrm{CO}_{2}$ no cultivo. A maior densidade celular obtida nas diferentes linhagens foi depois de 5min, exceto para as linhagens Chaetoceros gracilis e Nannochloris maculata, as quais alcançam maiores densidades aos 10 e 15min, respectivamente. A Variação do pH tendeu a ser ácido, na gama de 8 a 4, sem alterar a densidade de células, no entanto, os cultivos permaneceram livre de contaminantes. Em conclusão, estes resultados permitem estabelecer tempos adequados para a injeção de $\mathrm{CO}_{2}$, que reforçam a fase de crescimento exponencial acrescentando a densidade populacional num $30 \%$ sobre o estabelecido nesta fase.

Palabras-chave: microalgas, densidade celular, $\mathrm{CO}_{2}$, cultivo massivo.

\section{Introducción}

El calentamiento global es un gran y serio problema ambiental, como consecuencia principalmente de los gases del efecto invernadero como el metano, ozono troposférico, clorofluorocarbonatos y principalmente el dióxido de carbono $\left(\mathrm{CO}_{2}\right)$, que en los últimos años la concentración en la atmósfera ha ido en aumento, convirtiéndose así, en uno de los principales gases que componen el efecto invernadero (GEI), por ello su mitigación y utilización se ha estudiado de manera considerable [1].

En la actualidad, la reducción del uso de combustibles fósiles, la promoción de captura y secuestro del $\mathrm{CO}_{2}$ (mitigación biológica $\mathrm{O}$ biofijación) parecen ser la forma más viable de paliación [2]. La mitigación biológica, que consiste en convertirlos en materia orgánica $[3,4]$, se consigue a través de la fotosíntesis de las plantas terrestres y un enorme número de microorganismos fotosintéticos. Se sabe, que las plantas contribuyen con una reducción de sólo el $3-6 \%$ de las emisiones globales [3]. Sin embargo, las microalgas que representan alrededor del $0,5 \%$ de la biomasa global, presentan ventajas tales como altas tasas de crecimiento, eficiencia fotosintética y elevada producción de biomasa comparada con otros cultivos terrestres $[5,6]$ pueden llegar a producir alrededor del $70 \%$ del oxígeno neto en la tierra, por lo que se ha considerado de 10 a 50 veces más eficientes en fijación de $\mathrm{CO}_{2}$ que las plantas terrestres [1,7-11]. Los cultivos de microalgas han sido propuestos desde hace más de cincuenta años como fuente de combustibles renovables para reducir el efecto del calentamiento global [12]. Esto motivó que desde mediados de los años 70 el Departamento de Energía de los Estados Unidos de América (DOE) financiase proyectos de investigación, centrados en la producción de combustibles a partir de microalgas, comenzando con la producción de microalgas y su posterior transformación en biogás y biodiésel, también se ha financiado una red internacional sobre biofijación de $\mathrm{CO}_{2}$ con microalgas [13-15] y se han financiado proyectos basados en el empleo de fotobiorreactores cerrados [16] incluyendo el empleo de fibra óptica [17].

En Europa, se financiaron proyectos de investigación como el "Aquafuels" 0 el "Enerbioalgae". Mediante el primero se elaborar 
informes de las diversas iniciativas europeas llevadas a cabo en materia de producción de biocombustibles de microalgas y se ha tenido en cuenta el análisis de ciclo de vida considerando aspectos medioambientales, económicos y de sostenibilidad. El proyecto "Enerbioalgae" tuvo como finalidad el aprovechamiento y depuración de aguas residuales, urbanas e industriales a la vez que la depuración de gases de combustión mediante microalgas. Otro proyecto fue "ALLGAS" coordinado por Aqualia S.A. implicó el acoplamiento entre el tratamiento de aguas residuales, la captura de $\mathrm{CO}_{2}$ de gases de combustión y la producción de biomasa de microalgas con fines energéticos, suponiendo uno de los mayores retos tecnológicos actuales en este campo. En Japón se llevó a cabo el proyecto "Research Innovative Technologies of the Earth" (RITE) sobre la biofijación de $\mathrm{CO}_{2}$ con microalgas $[18,19]$. También, en Japón, la "Mitsubishi Heavy Industries" ha financiado proyectos [20].

Muchas investigaciones, concluyen que las microalgas son las más adecuadas para la fijación de $\mathrm{CO}_{2}$, porque son los únicos organismos capaces de adaptarse a ambientes cambiantes de condiciones climáticas (temperatura, $\mathrm{pH}$, salinidad, etc.) pudiendo incluso soportar condiciones extremas y utilizando altas concentraciones de $\mathrm{CO}_{2}$, a diferencia de los cultivos vegetales terrestres. Las microalgas mejoran su productividad con el aumento de la concentración de $\mathrm{CO}_{2}$ por encima de la concentración atmosférica (hasta cierto límite) [21]. Ciertas investigaciones han demostrado que las microalgas pueden fijar $\mathrm{CO}_{2}$ de lugares con concentraciones 1000 veces más altas que las ambientales, lo cual podría ser útil para controlar los altos niveles de $\mathrm{CO}_{2}$ industriales [22]. Sin embargo, la tasa de inyección y la transferencia eficiente del $\mathrm{CO}_{2}$ al medio de cultivo junto con la temperatura y la intensidad de luz son los mayores factores que influyen en la asimilación fotosintética del carbono $[23,24]$.

Al realizar cultivos solo con el $\mathrm{CO}_{2}$ presente en el aire $(0,036 \% \mathrm{v})$ se obtienen bajas productividades, por lo tanto la solución a este déficit es inyectar $\mathrm{CO}_{2}$, para obtener un buen crecimiento [25-30]. Los estudios realizados con diferentes microalgas, evidencian la eficiencia de captura de $\mathrm{CO}_{2}$, hasta del $80 \%$ a $99 \%$, que pueden alcanzarse en condiciones óptimas y tiempos cortos de residencia del gas, pero puede variar según el estado fisiológico [31]. Se ha reportado para algunas especies, tolerancia a concentraciones máximas de $\mathrm{CO}_{2}$
(\%), por ejemplo: Cyanidium celdanum (100\%), Scenedesmus sp. (80\%), Chlorococcum littorale (60\%), Synechococcus elongates (60\%), Euglena gracilis (45\%), Chlorella sp. (40\%), Eudorine sp. (20\%), Dunaliella tertiolecta (15\%), Nannochloris sp. (15\%), Chlamydomonas sp. (15\%), Tetraselmis sp. $(14 \%)$ y otras del genero Nannochloropsis, Chlorella, Scenedesmus, Phaedodactylum, Synechocystis y Spirulina, también mostraron tolerancia hasta un $40 \%[9,32-34]$.

Sin embargo, para la especies citadas anteriormente, los mejores rendimientos y productividades se han obtenido con la inyección directa de $5 \circ 15 \%$ v de $\mathrm{CO}_{2}$ a una velocidad de $0,025 \mathrm{vvm}$ (volumen de aire por volumen de trabajo por tiempo), lo óptimo es realizar inyecciones a bajas concentraciones y cortos intervalos de tiempo (por ejemplo $6 \mathrm{~g} / 100 \mathrm{~mL}$ cada $15 \mathrm{~min}$ ), o inyectar altas concentraciones pero a intervalos de tiempo mayores $(18 \mathrm{~g} / 100 \mathrm{~mL}$ cada $60 \mathrm{~min})[35,36]$; bajo estas condiciones se han logrado incrementar la productividad de biomasa en un $30 \%$ y los rendimientos de biomasa hasta tres veces más $[37,38]$.

Actualmente, se siguen impulsando proyectos dirigidos a utilizar microalgas para la eliminación de $\mathrm{CO}_{2}$ de gases de combustión, además se busca optimizar la velocidad de fijación de $\mathrm{CO}_{2}$ mediante selección de cepas adecuadas y la inyección mezclada con el aire. En países en desarrollo como el Perú, se están dando los primeros pasos para una producción masiva de microalgas con fines energéticos (obtención de biodiésel) y alimentarios (obtención de proteínas), sin embargo existen escasos estudios en los cuales se evalúe el efecto del $\mathrm{CO}_{2}$ sobre el crecimiento poblacional de las microalgas. Por lo cual, el objetivo del trabajo fue evaluar la cantidad y tiempo adecuado de ingreso de $\mathrm{CO}_{2}$ a seis cepas de microalgas marinas, con la finalidad de incrementar la densidad celular.

\section{Materiales y métodos}

\section{Microorganismos seleccionados y métodos de cultivo}

Se emplearon seis cepas de microalgas, de cepas nativas del Perú, Isochrysis galbana IMP-LBA-011 y Nannochloropsis spp. IMP-LBA-009 y cepas cosmopolitas como Nannochloropsis oceánica (Np), Chaetoceros gracilis (Chg), Phaeodactylum tricornutum (Ph) y Nannochloris maculata $(\mathrm{Na})$. Las cuales fueron proporcionadas por el Banco de Germoplasma de Organismos Acuáticos y el 
Laboratorio de cultivo de microalgas del Instituto del Mar del Perú (IMARPE).

Se realizaron cultivos masivos en el Laboratorio de Invernadero y Sala de procesos del área de Biotecnología Acuática, cultivos tipo batch de $300 \mathrm{~L}$, en tanques de fibra de vidrio $(100$ y $80 \mathrm{~cm}$ de diámetros mayor y menor, respectivamente; $62 \mathrm{~cm}$ de altura). Los cultivos fueron sometidos a condiciones semicontroladas del invernadero (se controlaron la aireación e inyección de $\mathrm{CO}_{2}$ ), en diferentes meses durante los años 2010 y 2011, los tanques fueron acoplados a un sistema de aireación por burbujeo y $\mathrm{CO}_{2}$ por separado, el tiempo de cultivo fue de tres días, asegurándose que los cultivos estén en fase exponencial del crecimiento microalgal. Diariamente, y para cada tanque, se realizó la toma de parámetros abióticos de temperatura y $\mathrm{pH}$, a las 8:00, 12:00 y 16:00 horas, utilizando un equipo multiparámetro (WTW 350i). Además, se tomaron los datos de temperatura y luminosidad del invernadero (termómetro de pared mínima y máxima Control Company y luxómetro Control Company, para cada parámetro, respectivamente).

Con el fin de aumentar la densidad celular, se probaron diferentes tiempos de inyección de $\mathrm{CO}_{2}(\mathrm{TI} \mathrm{CO})$ con un flujo continuo de $15 \mathrm{~L} / \mathrm{min}$ $(100 \mathrm{mg} / \mathrm{mL})$, proporcionado por un tanque de $\mathrm{CO}_{2}$ industrial de $30 \mathrm{~kg}(99,9999 \%$ de pureza). Se realizaron como mínimo tres pruebas por cada cepa, los tiempos de inyección evaluados fueron 0 (Control), 5, 10, 15, 20 y 25 minutos, tres veces al día (8:00, 12:00 y 16:00 horas), luego de tomar los parámetros abióticos. Se cuantificó diariamente la densidad celular y se observó la calidad de los cultivos utilizando una cámara de Neubauer bajo un microscopio óptico (marca LEITZ).

\section{Análisis de datos}

Los parámetros abióticos (Temperatura $\left({ }^{\circ} \mathrm{C}\right.$ ) y Luminosidad $\left(\mathrm{Lux} / \mathrm{m}^{2}\right)$ ) de las condiciones ambientales del invernadero, y los parámetros abióticos de los cultivos (Temperatura $\left({ }^{\circ} \mathrm{C}\right.$ ) y $\mathrm{pH}$ ), fueron evaluados por promedios simples y desviación estándar, con la finalidad de determinar su variabilidad y los posibles efectos sobre los cultivos microalgales.

Los datos del conteo celular (Densidad celular) fueron relacionados con los días de cultivo, mediante la ecuación de una regresión lineal, de los cuales se obtuvieron los valores de las pendientes de crecimiento (PCC). El parámetro pendiente, es el razón entre el incremento de $\mathrm{Y}$ y el incremento de $\mathrm{X}$ de la curva, nos permitió relacionar el tiempo con el número de microorganismos en la muestra.

\section{Análisis estadístico}

Se evaluó la normalidad de los datos mediante la prueba de Kolmogorov Sirmov, y la homogeneidad de varianza mediante la prueba de Cochran. Para el análisis de los promedios simples de Temperatura y $\mathrm{pH}$ se utilizó análisis de la varianza (ANDEVA), y test de Tukey. Se consideró un nivel de significancia de 0,05 para todas las pruebas y se utilizaron los programas estadísticos Minitab 16 (Minitab ${ }^{\circledR}$ Statistical Software, Pennsylvania, USA) y Systat 12 (Systat Software, San Jose, CA) para Windows 7. Se realizó una regresión lineal a los datos diarios de conteo celular, las pendientes nos indicaron la tendencia del crecimiento, posterior a ello, se correlacionó PCC de las ecuaciones halladas con los $\mathrm{TI} \mathrm{CO}_{2}$, para lo cual se usó la hoja de cálculo EXCEL 2010.

\section{Resultados y Discusión}

\section{Acondicionamiento y cultivo masivo}

El cultivo masivo de las diferentes cepas mantiene el mismo nivel de respuesta con relación a Temperatura y Luminosidad dentro del invernadero, independiente del tiempo en que fueron cultivados. La temperatura dentro del invernadero, tuvo un rango de variación de 31 a $39^{\circ} \mathrm{C}$. Mientras, el flujo de intensidad lumínica tuvo un rango 2158 a $26353 \mathrm{lux} / \mathrm{m}^{2}$, se presentan los promedios en la Tabla 1.

Respecto alos cultivos, no se observaron variaciones significativas en las variables de Temperatura y $\mathrm{pH}$ ( $p=0,09$ y $p=0,134$, respectivamente), el rango de variación fue $7,6-8,7$ para $\mathrm{pH}$ y $21,1-31,0^{\circ} \mathrm{C}$ para Temperatura. La variación de $\mathrm{pH}$, durante la inyección de $\mathrm{CO}_{2}$, tendió hacia la acidez, en un rango de 8,7 a 4,0 como mínimo en un lapso de 15 minutos para luego retornar a los valores promedios (entre 7 y 9). Esta variación no alteró el desarrollo celular, por el contrario, los cultivos permanecieron libres de contaminantes (protozoarios). 
Tabla 1. Promedio y desviación estándar de los parámetros abióticos del invernadero y meses durante los cuales fueron realizados los experimentos, para las diferentes cepas.

\begin{tabular}{lccl}
\hline Cepa Microalgal & Temperatura $\left({ }^{\circ} \mathbf{C}\right)$ & Luminosidad $\left(\mathbf{l u x} / \mathbf{m}^{2}\right)$ & Mes de cultivo \\
\hline IMP-LBM 009 & $37,8 \pm 2,6$ & $26046 \pm 6114$ & Febrero \\
$\mathrm{Na}$ & $35,2 \pm 2,3$ & $25689 \pm 2158$ & Mayo \\
$\mathrm{Np}$ & $38,2 \pm 3,0$ & $26353 \pm 5655$ & Febrero y marzo \\
$\mathrm{Ph}$ & $34,4 \pm 1,4$ & $16081 \pm 4589$ & Mayo \\
IMP-LBA-011 & $34,2 \pm 1,9$ & $15696 \pm 4683$ & Febrero \\
Chg & $31,6 \pm 1,6$ & $24426 \pm 5215$ & Diciembre \\
\hline
\end{tabular}

\section{Impacto del $\mathrm{CO}_{2}$ sobre los cultivos microalgales}

Se observa una relación inversa, ya que la PPC, disminuye a medida que aumenta el $\mathrm{TI} \mathrm{CO}_{2}$, de acuerdo a la curva sigmoidal de orden 3 (regresión polinómica) (Figuras 1 y 2).

Las cepas Nannochloropsis spp. IMPLBA-009, Isochrysis galbana IMPLBA-011, Nannochloropsis oceánica (Np) y
Phaeodactylum tricornutum (Ph), tienen una mayor pendiente de crecimiento sometidos a 5 min de inyección de $\mathrm{CO}_{2}$, logrando triplicar y hasta quintuplicar los valores de la pendiente, en el caso de Nannochloropsis spp. IMPLBA-009 y Phaeodactylum tricornutum (Ph), respectivamente, mejorando la densidad celular hasta un $30 \%$ (Figura 1a, 1b, 1c y 1d, respectivamente).
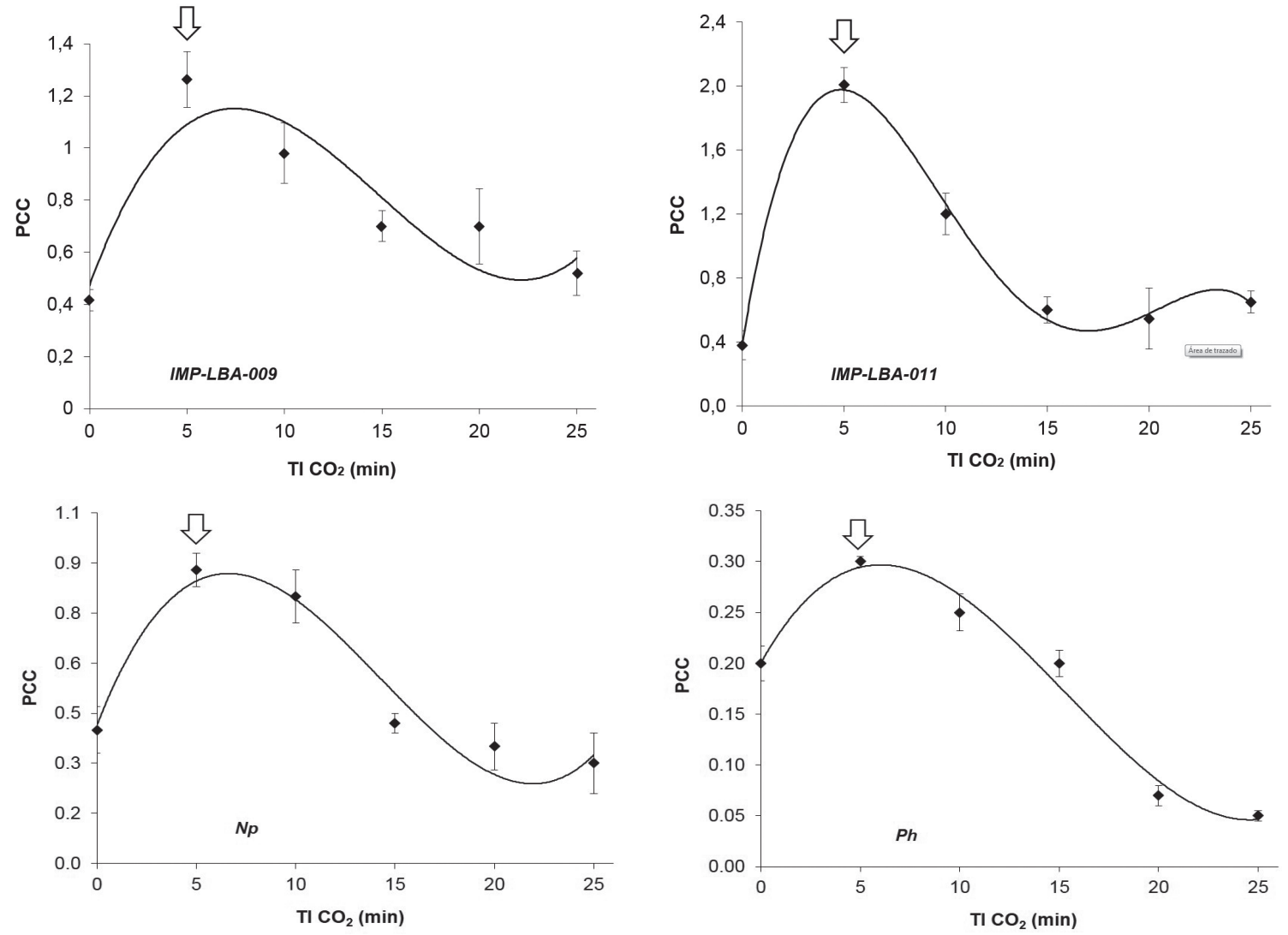

Figura 1. Relación entre tiempo de inyección de $\mathrm{CO}_{2}\left(\mathrm{TI} \mathrm{CO}_{2}\right)(\mathrm{min})$ y la pendiente de la regresión lineal del conteo celular (PCC) (cel/mL), para las diferentes cepas (líneas verticales son la desviación estándar de la pendiente de la regresión lineal (PCC)). 
A diferencia de las cepas Nannochloris maculata (Na) y Chaetoceros gracilis (Chg), las cuales obtienen la mayor pendiente en los 10 y $15 \mathrm{~min}$ respectivamente, duplicando los valores de las pendientes, con lo cual se mejora la densidad celular hasta un 20\% (Figura 2a y 2b, respectivamente).
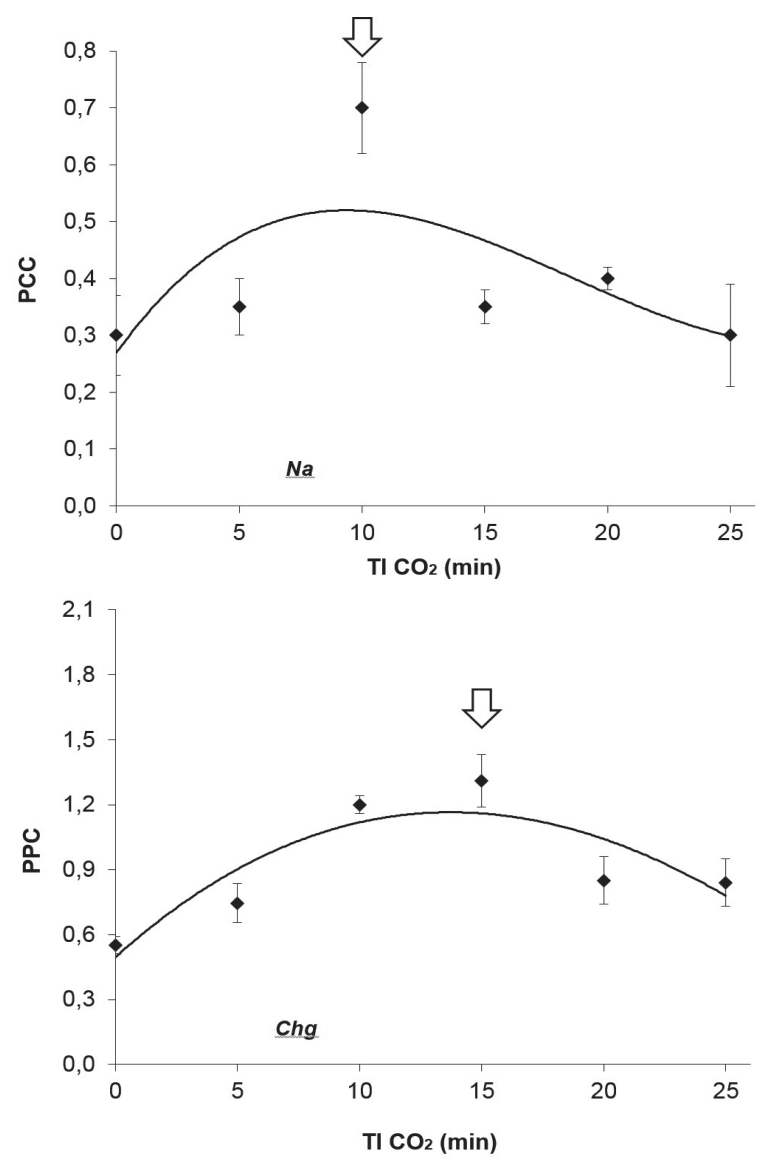

Figura 2. Relación entre tiempo de inyección de $\mathrm{CO}_{2}$ $\left(\mathrm{TI} \mathrm{CO}_{2}\right)(\mathrm{min})$ y la pendiente de la regresión lineal del conteo celular ( PPC) (cel/mL), a) cepa $\mathrm{Na} \mathrm{y} \mathrm{b)} \mathrm{cepa}$ Chg (líneas verticales son la desviación estándar de la pendiente de la regresión lineal (PCC)).

El suministro en masa del carbono a los sistemas de cultivo son las principales dificultades y limitaciones que deben ser resueltos [39]. El punto de todas las consideraciones, a tener en cuenta, es que el $\mathrm{CO}_{2}$ no debe llegar a la concentración superior que produce la inhibición, y por otra parte, nunca debe caer por debajo de la concentración mínima que limita el crecimiento. Sin embargo, estas concentraciones máximas (inhibición) y mínimas (limitación) varían de una especie a otra y no se conocen adecuadamente [40-42].

Roncarati et al. [43] señalan la importancia de suministrar el $\mathrm{CO}_{2}$ a los cultivos en cantidades adecuadas, ya que es un donador potencial de átomos de carbono, necesarios para la síntesis de compuestos orgánicos y que se refleja también en el perfil bioquímico. Para obtener altas productividades en el cultivo de microalgas, el suministro de $\mathrm{CO}_{2}$ es sumamente importante, ya que contrario a las plantas, el $\mathrm{CO}_{2}$ atmosférico no satisface los requerimientos necesarios para su crecimiento. La concentración de $\mathrm{CO}_{2}$ en la atmósfera es de $0,03-0,06 \%(0,36 \mathrm{mg} / \mathrm{ml})$ y la mayoría de las microalgas pueden tolerar niveles más elevados de $\mathrm{CO}_{2}$, por lo general hasta $150 \mathrm{mg} / \mathrm{ml}[44]$.

De acuerdo a los resultados de este estudio, se observa que la pendiente (PCC) de la regresión lineal es inversamente proporcional al tiempo de inyección de $\mathrm{CO}_{2}\left(\mathrm{TI} \mathrm{CO}_{2}\right)$. La mayor pendiente de crecimiento en las diferentes cepas, se obtuvo entre los 5 y 15 minutos. Lo que indicaría que no se necesita excesos de $\mathrm{CO}_{2}$ dentro del cultivo, es muy probable que las microalgas fijen o usen únicamente cierta cantidad de $\mathrm{CO}_{2}$, se puede pensar que no es tan alto. Además, se encontró una incidencia muy positiva al inyectar $\mathrm{CO}_{2}$ puro en un cultivo de microalgas; se observa que en un periodo corto de solo 3 días se duplicó, triplicó y hasta quintuplicó la pendiente de crecimiento en prácticamente todos los cultivos, en comparación con los cultivos sin inyección de $\mathrm{CO}_{2}$. Entonces, al aumentar la pendiente, aumentará la productividad, como se ha demostrado a escala experimental en tratamientos de aguas residuales, un aumento de más del doble [45-48]. Aunque, se ha comprobado que las microalgas desarrollan sus mayores tasas de crecimiento entre 1 y $5 \%$ de $\mathrm{CO}_{2}$ y que concentraciones por encima del $5 \%$ afectarían a su velocidad de crecimiento [49,50].

Ciertas especies experimentan sus crecimientos óptimos por encima del $5 \%$ de $\mathrm{CO}_{2}$, como por ejemplo Chlorella sp. RK-1 que lo logra al 10\% [51] o Chlorococcum littorale, al 20\% [52]. Pero también se ha reportado que Chlorella $s p$. se puede cultivar bajo $40 \%$ de $\mathrm{CO}_{2}$ [53]. Además, Maeda et al. [54] encontró una cepa de Chlorella sp. T-1 que podría crecer en $100 \%$ de $\mathrm{CO}_{2}$, aunque la tasa de crecimiento máxima se produjo bajo una concentración de $10 \%$. Otra cepa de Chlorella sp. puede crecer en cultivos enriquecidos con $\mathrm{CO}_{2}$ al $10 \%, 40 \%$ y $100 \%$, pero la tasa máxima de crecimiento, también fue en la concentración de 10\% [54-56]. De igual manera Scenedesmus sp. podría crecer en cultivos enriquecidos hasta con $80 \%$ de $\mathrm{CO}_{2}$ [53], sin embargo las mejores biomasas 
celulares se dan con el $20 \%$ de concentración de $\mathrm{CO}_{2}$. Chlorococcum littorale podría crecer bajo $60 \%$ de $\mathrm{CO}_{2}$ utilizando la técnica de la adaptación gradual [57]. Cepas del genero Caldarium y Cyanidium [58] y algunas otras especies de Cyanidium pueden crecer en $\mathrm{CO}_{2}$ puro [59].

Hoy en día, en una instalación real de biofijación de $\mathrm{CO}_{2}$ con microorganismos fotótrofos no sería necesario ni rentable trabajar con altas inyecciones de $\mathrm{CO}_{2}$ que acidifiquen el medio y que requieran especies específicas de ambientes ácidos, sino que se operará en las condiciones que normalmente favorecen el crecimiento del microorganismo. Porque, de acuerdo a Gómez y James [60], la fijación de $\mathrm{CO}_{2}$ dentro del cultivo no depende de los tiempos de inyección de éste, ya que se comprobó que no necesariamente inyectar $\mathrm{CO}_{2}$ en exceso o constantemente en pequeñas concentraciones implica que este sea captado en su totalidad por los microorganismos.

Se comprobó también que a pesar del potencial para acidificar el medio que tiene el $\mathrm{CO}_{2}$ inyectado puro, el $\mathrm{pH}$ alcanza un equilibrio en el cultivo, sin llegar a ser perjudicial para las microalgas, demostrando que pueden tolerar altas concentraciones de $\mathrm{CO}_{2}$, lo que se debe al ajuste anatómico estructural y redistribución de ciertos orgánulos celulares que pueden realizar [22]. Por ejemplo, algunas microalgas cultivadas solo con aireación constante (baja concentración de $\mathrm{CO}_{2}$ ) se observa los pirenoides con muchos más gránulos de almidón, que aquellas cultivadas con aire enriquecido con $\mathrm{CO}_{2}$, también se ha observado que los cloroplastos se encuentran cerca de la membrana plasmática y con envoltura eléctrica más densa, a diferencia de las cultivadas con más $\mathrm{CO}_{2}$, donde los cloroplastos se encuentran en la zona central, y es la membrana plasmática que tiene efecto eléctrico más denso [22]. Otros organismos en similares condiciones no podrían sobrevivir, por ejemplo Euglena gracilis, que fue cultivada en dos concentraciones de $\mathrm{CO}_{2}$, se observó el mayor crecimiento con la concentración de $5 \%$ y no hubo crecimiento en $45 \%$ [61], estas características explicarían porque durante los cultivos realizados no se observó la presencia de ningún contaminante (Protozoarios). Entre los principales factores que influyen el desarrollo microalgal, está la temperatura e iluminación, los cuales tienen un efecto sobre la actividad fotosintética, el contenido de pigmentos, velocidad de crecimiento y composición química de la microalga [62]. En las pruebas realizadas en condiciones de invernadero por un periodo de tres días y en diferentes tiempos, se observa que las condiciones abióticas tanto del invernadero (Temperatura y Luminosidad) y de los cultivos (Temperatura y $\mathrm{pH}$ ), cuyos datos fueron tomados previos a la inyección del $\mathrm{CO}_{2}$, no existen diferencias significativas, lo cual indica que no afectarían al desarrollo de las microalgas.

Como lo demuestran los estudios por ejemplo, Raghavan et al. [63] evaluaron el efecto de la temperatura y la salinidad sobre el crecimiento y composición de la diatomea Chaetoceros calcitrans, no evidenciaron variación en el crecimiento celular, pero si en el contenido lipídico, que fue mayor a temperaturas entre 20 y $25^{\circ} \mathrm{C}$ y que disminuyó al aumentar la salinidad. Converti et al. [64], realizaron experimentos con Nannochloropsis oculata y Chlorella vulgaris, demostrando, a temperaturas superiores a los $35^{\circ} \mathrm{C}$ se vio una disminución en la tasa de crecimiento de las microalgas, pero a temperaturas en rangos de 30 y $15^{\circ} \mathrm{C}$, no se observa este efecto, pero el contenido de lípidos varía entre especies. Y otras como del género Scenedesmus crecen mejor en el rango de 25 a $35^{\circ} \mathrm{C}$, algunas mostrando sus óptimos incluso a $40^{\circ} \mathrm{C}[53,54,65-67]$.

Al considerar los resultados de los estudios descritos anteriormente, parecería que las concentraciones elevadas de $\mathrm{CO}_{2}$ no son perjudiciales para el cultivo microalgal. De hecho, sugiere que los enormes aumentos en la concentración de $\mathrm{CO}_{2}$ a veces pueden conducir a un enorme aumento en el crecimiento de las microalgas. A los efectos de la captura de $\mathrm{CO}_{2}$, el uso de microalgas es una tecnología única. De hecho las microalgas están en la parte superior de las opciones por su excepcional eficiencia en la conversión de energía, el tamaño de eliminación de $\mathrm{CO}_{2}$ y la utilidad de sus subproductos $[68,69]$. La tecnología de producción de energía alternativa, que hoy en día es respetuoso del medio ambiente, trabaja bajo concentraciones limitadas de $\mathrm{O}_{2}$, y una amplia gama de condiciones térmicas y de luz, solo es necesario seleccionar el tipo adecuado de microalgas y luego adaptarlas a concentraciones adecuadas de $\mathrm{CO}_{2}$ para tener el potencial de producir subproductos útiles.

\section{Conclusión}

La regulación de la cantidad de carbono suministrado afecta positivamente la densidad celular. De acuerdo a la corta experiencia de estas pruebas y los periodos estacionales dentro de los 
cuales se han llevado a cabo, han demostrado que el rango de inyección de $\mathrm{CO}_{2}$ para nuestros cultivos y condiciones de trabajo, tiene que estar dentro de los 5 y 15min de inyección, en tres raciones (mañana, mediodía y tarde), con lo cual se podrá mejorar hasta un $30 \%$ en el crecimiento celular. Estos resultados representan un avance importante en la producción de cultivos microalgales, ya que abre las posibilidades para mejorar la producción total de biomasa mientras se reducen costos operacionales y tiempos de producción, además permite desarrollar estrategias para acoplar la producción de diferentes metabolitos (ya sea para la obtención de biocombustibles como biodiésel u otros elementos) con la captura de $\mathrm{CO}_{2}$.

\section{Referencias bibliográficas}

[1] Ferreira L, Rodrigues M, Converti A, Sato S, Carvalho J. Arthrospira platensis (Spirulina) cultivation in tubular photobioreactor: Use of no-cost $\mathrm{CO}_{2}$ from ethanol fermentation. Appl. Energy. 2012;92:379-85.

[2] Wackett L. Microbial-based fuels: science and technology. Microb Biotechnol. 2008;1:211-25.

[3] Skjanes K, Lindblad P, Muller J. $\mathrm{BiOCO}_{2}-\mathrm{a}$ multidisciplinary, biological approach using solar energy to capture $\mathrm{CO}_{2}$ while producing $\mathrm{H}_{2}$ and high value products. Biomol Eng. 2007;24:405-13.

[4] Pulz O, Gross W. Valuable products from biotechnology of microalgae. Appl Microbiol Biotechnol. 2004;65:635-48.

[5] Um B, Kim Y. Review: A change for Korea to advance algal-biodiesel technology. J. Ind. Eng. Chem. 2009;15(1):1-7.

[6] Šoštarie M, Golob J, Bricelj M, Klinar D, Pivec A. Studies on the growth of Chlorella vulgaris in culture media with different carbon sources. Chem. Biochem. Eng. 2009;23(4):471-7.

[7] Costa J, Linde G, Atala D, Mibielli G. Modelling of growth conditions for cyanobacterium Spirulina platensis in microcosms. World $\mathrm{J}$. Microbiol Biotechnol. 2000;16(1):15-8.

[8] Crutzen P, Mosier A, Smith K, Winiwarter W. $\mathrm{N}_{2} \mathrm{O}$ release from agro-biofuel production negates global warming reduction by replacing fossil fuels. Atmos. Chem. Phys. 2008;8:389-95.

[9] De Morais M, Costa J. Biofixation of carbon dioxide by Spirulina sp. and Scenedesmus obliquus cultivated in a three-stage serial tubular photobioreactor. J. Biotechnol, 2007;129:439-45.
[10] Jeong L, Gillis J, Hwang J. Carbon dioxide mitigation by microalgal photosynthesis. Bull. Korean. Chem. Soc. 2003;24(12):1763-6.

[11] Hughes E, Benemann J. Biological fossil $\mathrm{CO}_{2}$ mitigation. Energy. Convers. Manage. 1997;38:467-73.

[12] Oswald W, Golueke C. Biological transformation of solar energy. Adv. Appl. Microbiol. 1960;11:223262.

[13]Benemann JR, Weissman JC, Koopman BL, Oswald WJ. Energy production by microbial photosynthesis. Nature. 1977;268(5615):19-23.

[14] Sheehan J, Dunahay T, Benemann J, Roessler P. A look back at the US Department of Energy's aquatic species program-Biodiesel from algae (NREL/TP-580-24190). Golden, CO: National Renewable Energy Laboratory (NREL), US DOE;1998.

[15]Benemann J. Biofixation of $\mathrm{CO}_{2}$ and greenhouse gas abatement with algae technology roadmap. Report No. 7010000926. Morgantown, United States: Prepared for the U.S. Department of Energy National Energy Technology Laboratory; 2003.

[16]National Energy Technology Laboratory. Recovery and sequestration of $\mathrm{CO}_{2}$ from stationary combustion systems by photosynthesis of microalgae. Pittsburgh, Estados Unidos: Nakamura T; 2004.

[17] Bayless, D, Kremer, G, Vis-Chiasson, M, Stuart, $\mathrm{B}$, Shi, L. Photosynthetic $\mathrm{CO}_{2}$ Mitigation Using a Novel Membrane-Based Photobioreactor. J. Environ. Eng. Manag. 2006;16(4):209-15.

[18] Usui $N$, Ikenouchi $M$. The biological $\mathrm{CO}_{2}$ fixation and utilization project by RITE (1) - highly effective photobioreactor systems. Energy Conserv. Mgmt. 1996;38:S487-92

[19] Ikuta Y, Weissman J. Carbon dioxide utilizationmicroalgae. Technology. 2000;75:137-45.

[20] Nakajima Y, Ueda R. The effect of reducing light-harvesting pigment on marine microalgal productivity. J. App. Phycol. 2000;12:285-90.

[21] Matsumoto $H$, Hamasaki $A$, Sioji N, Ikuta $Y$. Influence of $\mathrm{CO}_{2}, \mathrm{SO}_{2}$, and $\mathrm{NO}$ in Flue Gas on Microalgae Productivity. J. Chem. Eng. 1997;30(4):620-24.

[22] Papazi A, Makridis P, Divanach P, Kotzabasis $\mathrm{K}$. Bioenergetic changes in the microalgal photosynthetic apparatus by extremely high $\mathrm{CO}_{2}$ concentrations induce an intense biomass production. Physiol. Plant. 2008;132(3):338-49.

[23] Hodaifa G, Martinez M, Sanchez S. Daily doses of light in relation to the growth of 
Scenedesmus obliquus in diluted threephase olive mill wastewater. J. Chem Technol Biotechnol. 2009;84:1550-8.

[24] Pulz O. Photobioreactors: production systems for phototrophic microorganisms. Appl Microbiol Biotechnol. 2001;57(3):287-93.

[25] Fadhil S. Microalgae tolerance to high concentrations of carbon dioxide: A review. J. Environment. Protection. 2011;2:648-654.

[26] Chiu S, Kao C, Chen C, Kuan T, Ong S, Lin C. Reduction of $\mathrm{CO}_{2}$ by a high-density culture of Chlorella $s p$. In a semicontinuous photobioreactor. Bioresour. Technol. 2008;99(9):3389-96.

[27] Babcock R, Malda J, Radway J. Hydrodynamics and mass transfer in a tubular air-lift photobioreactor. J. Appl. Phycol. 2002;14:169-14.

[28] Morita M, Watanabe Y, Okawa T, Saiki H. Photosynthetic productivity of conical helical tubular photobioreactors incorporating Chlorella sp. under various culture medium flow conditions. Biotechnol. Bioeng. 2001;74(2):136-44.

[29] Merchuk J, Gluz M, Mukmenev I. Comparison of photobioreactors for cultivation of the red microalga Porphyridium sp. J. Chem. Technol. Biotechnol. 2000;75(12):1119-26.

[30] Lee $\mathrm{Y}$, Pirt S. $\mathrm{CO}_{2}$ absorption rate in an algal culture: effect of $\mathrm{pH}$. J. Chem. Tech. Biotechnol.1984;34(1):28-32.

[31] Keffer J, Kleinheinz G. Use of Chlorella vulgaris for $\mathrm{CO}_{2}$ mitigation in a photobioreactor. J. Ind. Microbiol. Biotechnol. 2002;29:275-80.

[32] Iwasaki I, Hu Q, Kurano N, Miyachi S. Effect of Extremely High- $\mathrm{CO}_{2}$ Stress on Energy Distribution Between Photosystem I and Photosystem II in a High- $\mathrm{CO}_{2}$ Tolerant Green Alga, Chlorococcum littorale and the Intolerant Green Alga Stichococcus bacillaris. J. Photochem. Photobiol. B. 1998;44(3):184-90.

[33] Murakami M, Ikenouchi M. The biological $\mathrm{CO}_{2}$ fixation and utilization by RITE (2) Screening and breeding of microalgae with high capability in fixing $\mathrm{CO}_{2}$. Energy Conv. Manag. 1997;38:S493-7.

[34] Vinod K. Feels algae are not yet ready for primetime?. Oilgae. Disponible en: http://www. oilgae.com/blog/2008/10/vinod-khosla-feelsalgae-arenot-yet.html. Acceso el 20 de octubre del 2014.

[35] Sierra E, Acien F, Fernadez J, Garcia J, Gonzalez C, Molina E. Characterization of a flat plate protobioreactor for the production of microlagae. Chem. Eng. J. 2008;138:136-147. [36]Zhang K, Kurano N, Miyachi S. Optimized aeration by carbon dioxide gas for microalgal production and mass transfer characterization in a vertical flat-plate photobioreactor. Bioproc. Biosyst. Eng. 2002;25:97-101.

[37] Jeong Y, Ishida K, Ito Y, Okada S, Murakami M. Bacillamide, a novel algicide from the marine bacterium, Bacillus sp. SY-1 against the harmful dinoflagellate, Cochlodinium polykrikoides. Tetrahedron Lett. 2001;4:8005-7.

[38] Douskova I, Doucha J, Livansky K, Machat J, Novak P, Umysova D, Zachleder V, Vitova M. Simultaneous flue gas bioremediation and reduction of microalgal biomass production costs. Appl. Microbiol. Biotechnol. 2009;82:179-85.

[39] Benemann J, Tillet D, Weissman J. Microalgae biotechnology. Trends in Biotechnology. 1987;5:47-53.

[40] Coleman J. The molecular and biochemical analyses of $\mathrm{CO}_{2}$-concentrating mechanisms in cyanobacteria and microalgae. Plant Cell Environ. 1991;14:861-7.

[41] Miller A, Espie G, Canvin D. Physiologicalaspects of $\mathrm{CO}_{2}$ and $\mathrm{HCO}_{3}$ - transport by cyanobacteria - a review. Can J Bot Rev Can Bot. 1990;68:1291-302.

[42] Badger M, Price G. Carbonic-anhydrase activity associated with the Cyanobacterium synechococcus PCC7942. Plant Physiol. 1989;89:51-60.

[43] Roncarati A, Meluzzi A, Acciarri S, Tallarico N, Melotti P. Fatty Acid of Different Microalgae Strains (Nannochloropsis sp., Nannochloropsis oculata (Droop) Hibberd, Nannochloropsis atomus Butcher and Isochrysis sp.) According to the culture Phase and the Carbon Dioxide Concentration. J. World Aquacult. Soc. 2004;35(3):401-11.

[44] Brennan L, Owende P. Biofuels from microalgae - A review of technologies for production, processing and extractions of biofuels and co-products. Renew. Sustainable Energy Rev. 2010;14:557-577.

[45]Mann G, Schlegel M, Schumann R, Sakalauskas A. Biogas-conditioning with microalgae. Agron. Res. 2009;7(1):33-8.

[46] Park J, Craggs R, Shilton A. Recycling algae to improve species control and harvest efficiency from a high rate algal pond. Water Res. 2011;45:6637-49.

[47] Martínez L. Eliminación de $\mathrm{CO}_{2}$ con microalgas autóctonas (Tesis Doctoral) León, España: 
Instituto de Recursos Naturales Universidad de León; 2008.

[48] Mendoza H, De la Jara A, Portillo E. Planta piloto de cultivo de microalgas: Desarrollo potencial de nuevas actividades económicas asociadas a la biotecnología en Canarias. España: Gráficas Tenerife S.A. 2011.

[49]Nielsen M. Photosynthetic characteristics of the Coccolithophorid Emiliania huxleyi (Prymnesiophyceae) exposed to elevated concentrations of dissolved inorganic carbon. J. Phycol. 1995;31:715-9.

[50]Myers J. Growth characteristics of algae in relation to the problems of mass culture. In: Algal culture from laboratory to pilot plant, J.S. Burlew (Ed.), Carnegie Institution of Washington, Washington, DC.1953.

[51] Sung K, Lee J, Shin C, Park S, Choi M. $\mathrm{CO}_{2}$ fixation by Chlorella sp. KR-1 and its cultural characteristics. Biores. Biotechnol. 1999;68:269-73.

[52] Kurano N, Ikemoto H, Miyashita H, Hasegawa $\mathrm{T}$, Hata $\mathrm{H}$, Miyachi S. Fixation and Utilization of Carbon Dioxide by Microalgal Photosynthesis. Energy Conversion and Management. 1995;36(6-9):689-92.

[53] Hanagata N, Takeuchi T, Fukuju Y. Tolerance of Microalgae to High $\mathrm{CO}_{2}$ and High Temperature. Phyto-chemistry. 1992;31(10):3345-8.

[54] Maeda K, Owada M, Kimura N, Omata L Karube I. $\mathrm{CO}_{2}$ Fixation from the Flue Gas on Coalfired Thermal Power Plant by Microalgae. Energ. Convers. Manage. 1995;36(6-9):71720.

[55] Hirata S, Taya M, Tone S. Characterization of Chlorella Cell Cultures in Batch and Continuos Operations undera Photoautotrophic Condition. J. Chem. Eng. of Japan. 1996a;29(6):953-9.

[56] Hirata S, Hayashitani M, Taya M, Tone S. Carbon Dioxide Fixation in Batch Culture of Chlorella sp. Using a Photobioreactior with a Sunlight-Collection Device. J. Mar. Biotechnol. 1996b;81(5)470-2.

[57] Kodama M, Ikemoto $H$, Miyachi S. A new species of highly $\mathrm{CO}_{2}$-tolreant fast-growing marine microalga suitable for high-density culture. J Mar Biotechnol. 1993;1:21-5.

[58] Seckbach J, Gross H, Nathan M. Growth and photosynthesis of Cyanidium caldarium cultured under pure $\mathrm{CO}_{2}$. Israel J. of Bot. 1971;20:84-90.
[59] Graham L, Wilcox L. Algae. Estados Unidos: Prentice-Hall, Inc; 2000.

[60] Gomez A, Jaimes N. Estudio de la incidencia del suministro de $\mathrm{CO}_{2}$ en el crecimiento de las microalgas en un fotobiorreactor a escala laboratorio (Proyecto de pregrado) Bucaramanga, Colombia: Universidad Industrial de Santander; 2010.

[61] Nakano Y, Miyatake K, Okuno H, Hamazaki K, Takenaka S, Honami N, Kiyota M, Aiga I, Kondo J. Growth of Photosynthetic Algae Euglena in High $\mathrm{CO}_{2}$ Conditions and Its Photosynthetic Characteristics. Acta Horticulturae. 1996;440(9):49-54.

[62] Renaud S, Zhou H, Parry D, Loung-Van T, Woo K. Effect of temperature on the growth, total lipid content and fatty acid composition of recently isolated tropical microalgae Isochrysis sp. Nitzschia closterium, Nitzschia paleacea, and commercial species Isochrysis sp. J. Appl. Phycol. 1995;7(6):595-602.

[63] Raghavan G, Haridevi C, Gopinathan C. Growth and proximate composition of the Chaetoceros calcitrans $f$. pumilus under different temperature, salinity and carbon dioxide levels. Aquacult. Res. 2008;39(10):1053-8.

[64] Converti A, Casazza A, Ortiz E, Perego P, Del Borghi M. Effect of temperature and nitrogen concentration on the growth and lipid content of Nannochloropsis oculata and Chlorella vulgaris for biodiesel production. Chemical Eng. and Process. 2009;48:1146-51.

[65] Chang E, Yang S. Microalgae for biofixation of carbon dioxide. Bot. Bull. Acad. Sin. 2003;44:4352.

[66] Watanabe Y, Ohmura N, Saiki H. Isolation and determination of cultural- characteristics of microalgae which functions under $\mathrm{CO}_{2}$ enriched atmosphere. Energ. Convers. Manage. 1992;33:545-52.

[67] Yue L, Chen W. Isolation and determination of cultural characteristics of a new highly $\mathrm{CO}_{2}$ tolerant fresh water microalgae. Energ Convers Manage. 2005;46:1868-76.

[68] Li Y, Horsman M, Wu N, Lan C, Dubois-Calero N.LiY, Horsman M, Wu N, Lan C, Dubois-Calero N. Biofuels from microalgae. Biotechnol Prog. 2008;24(4):815-20. ASAP Article, DOI 10.1021/ bp070371kS8756-7938(07)00371-2; 2008.

[69] Chisti Y. Biodiesel from microalgae. Biotechnol. Adv. 2007;25:294-306. 